\title{
Preparación de docentes en la atención educativa a escolares doblemente excepcionales
}

Preparation of teachers in the educational attention to doubly exceptional schoolchildren

Recepción del artículo: 20-07-2021 | Aceptación del artículo: 19-12-2021
Para referenciar este artículo:

Martínez-Rangel, A. Y., Castellanos-Simons, D. y López-Aymes, G. (2022). Preparación de

Docentes en la Atención Educativa a

Escolares Doblemente Excepcionales.

Revista ConCiencia EPG, 7(1), 15-35.

https://doi.org/10.32654/CONCIENCIAEP

G.7-1.2

Autora corresponsal: Doris Castellanos Simons dcastellanos@uaem.mx Morelos, México

\section{Resumen}

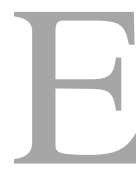

l objetivo del estudio que se reporta fue analizar el nivel de sensibilización y preparación de los maestros para detectar y brindar atención educativa a alumnos con "doble excepcionalidad" - Altas Capacidades (AC) y Trastorno por Déficit de Atención e Hiperactividad (TDAH), con el fin de determinar sus necesidades de orientación y capacitación en una escuela primaria del sector privado en Morelos.

Se desarrolló un estudio descriptivo, cualitativo, en el que participaron los docentes que impartían clases a escolares de una primaria de Xochitepec con diagnóstico previo de TDAH emitido por especialistas, y evaluación de AC mediante diferentes instrumentos. Con cada docente se desarrollaron entrevistas semiestructuradas para recolectar datos que permitieran identificar sus necesidades de preparación en el área. Derivado del diagnóstico de los participantes, se diseñó e implementó un programa de intervención. El proceso formativo se caracterizó por posibilitar una reflexión compartida respecto a la necesidad de brindar una atención educativa acorde a las características y necesidades de los alumnos, a partir de un enfoque basado en el reconocimiento de sus fortalezas para propiciar un ambiente desarrollador para todos los alumnos. Los docentes asumieron una postura autocrítica y tomaron conciencia de la trascendencia de su rol en el desarrollo del potencial de los alumnos. 
Palabras Clave: Alta capacidad, TDAH, doble excepcionalidad, sensibilización y preparación docente.

\section{Abstract}

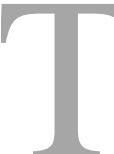

he objective of this study was to analyze the level of awareness and preparation of teachers to detect and provide educational attention to students with "double exceptionality" High Abilities (HA) and Attention Deficit Hyperactivity Disorder (ADHD), in order to determine their needs of orientation and training. A qualitative study was carried out in a private elementary school of Morelos. Participants were teachers of schoolchildren with previous diagnosis of ADHD issued by specialists and evaluated as highly able by means of different instruments. Semi-structured interviews were conducted with each teacher in order to identify their preparation/training needs in the area. Also, based on the diagnosis of the students, an intervention program was designed and implemented. The teachers training process allowed the reflection on the urgency to provide educational attention according to the characteristics and needs of the students, from an approach based on the recognition of their strengths, in order to generate enriched educational environments for them. Teachers assumed a self-critical position and became aware of the importance of their role in the development of students' potential.
Key Words: High abilities, Attention-Deficit Hyperactivity disorder (ADHD), Twice exceptional students, Teacher's training.

\section{Introducción}

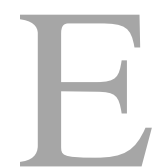

n la actualidad, uno de los temas más desafiantes en el sistema educativo mexicano, es el relativo a cómo convertir en realidad la aspiración de una escuela incluyente. La atención educativa a los alumnos con altas capacidades, aptitudes sobresalientes y/o talentos, se ha vuelto una preocupación cada vez más reconocida y extendida. El papel de los diferentes agentes educativos docentes, familias, directivos y los propios estudiantes- se vuelve un aspecto de gran relevancia. En especial, se reconoce la importancia de los docentes como piezas clave en las acciones que, desde la escuela, pretenden dar atención a esta población de estudiantes con necesidades educativas especiales.

El presente estudio se inserta en el marco de una investigación más amplia dirigida a preparar a los docentes para hacer frente a la detección y atención de niños con Altas Capacidades Intelectuales (ACI) que también presentan dificultades de aprendizaje, diagnosticados con Trastorno por Déficit de Atención e Hiperactividad (TDAH). El problema de investigación se centra en la complejidad que representa para los docentes, identificar y dar atención a estudiantes con condiciones que aparentemente son de naturaleza opuesta, la llamada doble 
excepcionalidad, entendida como la coexistencia de la alta capacidad intelectual con un diagnóstico de discapacidad o trastorno del aprendizaje, posibilitando un impacto directo en el desarrollo y aprendizaje de niños y adolescentes (Conejeros et al., 2018; Pardo, 2022, 2004, Valadez et al., 2018).

Las posibilidades de combinación en la dualidad son varias. Pardo de Santayana (2004) establece una categorización de cuatro grupos que contienen la superioridad intelectual en un extremo, y en el otro, una deficiencia que puede ser sensorial, motriz, cognitiva y dificultades de aprendizaje. La última combinación, según la autora, es la población doblemente excepcional porcentualmente mayor respecto a las otras opciones, siendo el TDAH, el diagnóstico de mayor prevalencia.

Los alumnos doblemente excepcionales que presentan la dualidad Alta Capacidad-TDAH, "poseen características similares a las de estudiantes con altas capacidades, como razonamiento abstracto superior, variedad de intereses, fluidez verbal $y$ alta creatividad. Sin embargo, simultáneamente presentan rasgos de inatención, hiperactividad e impulsividad" (Álvarez et al., 2019, p. 418).

Estos estudiantes pueden mostrarse poco receptivos $\mathrm{y}$ dedicados a las actividades, por lo que su rendimiento académico puede ser bajo, su nivel de ejecución no es proporcional con el potencial intelectual. Este fenómeno se conoce como "enmascaramiento", proceso que consiste en el ocultamiento entre condiciones (Pardo de Santayana, 2002).

Diversas investigaciones (Baum, 1989; Beckley, 1998; Pardo de Santayana, 2002; Pffeifer, 2015) señalan como consecuencia del enmascaramiento, la falta de diagnósticos pertinentes. Son alumnos adscritos a otra categoría de identificación: estudiantes superiores intelectualmente con bajo rendimiento; con dificultades de aprendizaje sin evidenciar su habilidad intelectual, y alumnos con habilidades que no han sido identificados en ninguna de las excepcionalidades. En cualquier caso, son infra-diagnosticados, en consecuencia, se manejan con respuestas educativas incompletas o erróneas. Sin embargo, lo que se requiere es comprender la simultaneidad como "una realidad compleja en la que un individuo demanda al mismo tiempo, una atención centrada en la dificultad y una respuesta creativa en relación con su potencialidad" (Pardo de Santayana, 2004, p. 38).

La omisión de las necesidades educativas de este tipo de alumnado puede propiciar secuelas negativas en su desarrollo académico, motivacional $\mathrm{y}$ socioemocional. En el contexto escolar, las actividades propias de la vida académica generan en ellos, una sensación de desfase que eventualmente se traduce en sentimientos de ansiedad y tensión. Diversos estudios de carácter cualitativo (Álvarez-Cárdenas et al., 2019; Gómez et al., 2016; Pfeiffer, 2015), señalan que los alumnos doblemente excepcionales presentan una noción de discrepancia de su condición a pesar de desconocerla: saben que poseen habilidad para procesar 
información pues comprenden las lecciones con relativa facilidad, pero se aburren rápido y les cuesta trabajo persistir en actividades más complejas. Por este motivo, les resulta frustrante no ser capaces de reflejar su nivel de habilidad; la frustración persistente los lleva a fracasar incluso en áreas donde los síntomas propios de la dualidad no inciden de manera directa.

Por otra parte, pueden manifestar características académicas, sociales, emocionales $\quad 0 \quad$ comportamentales específicas que deterioran su desenvolvimiento. Entre las características más destacables se señalan: una necesidad reiterativa de perfección, manejo deficiente de sus emociones, baja autoestima, sensación de inseguridad en su propio desempeño, marcada intolerancia a la frustración, disposición para rendirse ante tareas que representan un reto mayor y sentimientos constantes de baja eficacia. En general se manifiesta un autoconcepto con rasgos negativos, el cual es resultado de la constante sensación de fracaso. En las descripciones que hacen de sí mismos, generan expresiones fatalistas como "su cerebro extraño y estúpido" y hacen referencias al deseo que tienen de mejorar, pero su cabeza no lo permite (ÁlvarezCárdenas et al., 2019).

En el manejo emocional y su interacción con otros, se observan también dificultades importantes debido a la disincronía entre su madurez emocionalsocial y su alta inteligencia, generando consecuencias profundas en su proceso de integración social. Les cuesta trabajo relacionarse de manera equilibrada pues tienden a dominar y a generar sus propias reglas en el juego, lo que se traduce en un eventual rechazo. Presentan reacciones exageradas o no proporcionales, que se pueden tornar en manifestaciones groseras e incluso desafiantes. La sensación de rechazo y las percepciones negativas influyen directamente en su desempeño social y manejo emocional, asumen la responsabilidad del fracaso con cierto pesimismo y pueden desistir de todo esfuerzo (Pardo de Santayana, 2002).

Específicamente, en la relación con profesores, habitualmente tienen dificultades que se gestan a partir de la contraposición de las características de su comportamiento. Los profesores manifiestan confusión y atribuyen las deficiencias a problemas actitudinales y/o conductuales, las dificultades son interpretadas como una cuestión de desgano, sobre todo, de un bajo nivel de habilidad. Los actos impulsivos propios de la manifestación del TDAH, son interpretados como un rompimiento a las reglas (por ejemplo, interrumpir de manera frecuente sin respetar los turnos), como una forma de oposición a la figura de autoridad (respuestas inadecuadas, bromas o frases sarcásticas) y en general, como un no seguimiento de instrucciones permanente (pararse de lugar, moverse en el salón de clases). Adicionalmente, existe un marcado estereotipo sobre las ACI, pues se les asigna un atributo de perfección académica, esto impide que sean consideradas como una dificultad paralela en el aprendizaje (Luque-Parra, LuqueRojas y Hernández, 2017).

Constituye entonces todo un desafío que los docentes tomen conciencia de que, 
en este tipo de perfil, existe una alta capacidad enmascarada. La falta de conocimientos y sensibilización del profesorado sobre este tópico solo complica la situación, conduciendo a menudo a dificultades para comprenderlo y consecuentemente, brindar apoyo y acompañamiento al alumnado (Gómez et al., 2016).

El mayor riesgo de la invisibilización de esta dualidad es que la falta de recursos de los estudiantes para funcionar $y$ adaptarse a la dinámica escolar, después de un tiempo, se traduce en aislamiento, baja autoestima, rezago y afecciones psiquiátricas, inclusive un mayor riesgo de trastornos afectivos (García, 2013). Es indispensable evitar que pasen inadvertidos y que se mantengan doblemente invisibles.

La falta de identificación es un problema prioritario, representa una limitación para el desarrollo del potencial de innumerables niños. Partiendo del reconocimiento de que la doble excepcionalidad es una categoría que se deriva de las altas capacidades, es posible considerar las causas que explican la falta de detección y atención de los alumnos con altas capacidades, como una alternativa para entender la problemática de atención de la doble excepcionalidad. Las investigaciones de Castellanos (2014) señalan que, entre las principales razones en el contexto académico, se encuentran aquellas relacionadas con los docentes, con la falta de sensibilización y/o preparación, a veces con insuficiente motivación para incidir favorablemente en el desarrollo de estos alumnos $\mathrm{y}$, sobre todo, con sus insuficientes recursos teórico metodológicos para planificar, ejecutar, dar seguimiento y evaluar estrategias psicoeducativas de diagnóstico y atención diferenciada. En particular, autores como Conejeros et al. (2018), Gómez-Arizaga et al., (2016), Álvarez-Cárdenas et. al (2015), Pfeiffer (2015), entre otros, han destacado cómo los errores y problemáticas existentes en el diagnóstico de la doble excepcionalidad en el contexto escolar depende en gran medida de la preparación del profesorado, mientras otros estudios como el de Bianco et al. (2010) analizan el impacto de las actitudes de docentes hacia la discapacidad como un factor significativo en el reconocimiento de los estudiantes con altas capacidades con este tipo de condiciones.

Es necesario, entonces, preparar a las (los) docentes en el reconocimiento de esas características diferenciales y en la creación de propuestas de intervención basadas en los perfiles de fortalezas de este alumnado. El objetivo del estudio que se reporta fue precisamente, analizar el nivel de sensibilización y preparación de los maestros en el campo de la atención educativa a los alumnos con $\mathrm{AC}$, y en particular, en el tema de la "doble excepcionalidad", para determinar sus necesidades de orientación y capacitación.

\section{Método}

Diseño: se desarrolló un estudio descriptivo, con un enfoque cualitativo, que buscó indagar en la propia experiencia de los docentes, de sus necesidades en este campo, y en su comprensión de la problemática (Flick, 2012). 
Participantes: Por la naturaleza del proyecto general, los participantes fueron las/los docentes responsables de impartir clases a seis estudiantes de la escuela (de los grados 1ro a 6to) que fueron previamente identificados con doble excepcionalidad (AC-TDAH) a partir de un conjunto de criterios como la existencia de un diagnóstico establecido por un especialista, y los resultados en la Prueba de
Matrices Progresivas, Escala Coloreada (Raven, Court \& Raven, 2001) y en la Escala de Inteligencia de Wechsler para niños (WISC-IV, 2007). Participaron seis maestras de educación regular, 1 maestra de Computación, 1 maestra de Educación Física, y 1 maestro de Educación Artística (Tabla 1), que manifestaron su disposición e interés de participar en el proyecto, firmando un consentimiento informado.

\section{Tabla 1}

Datos de los docentes participantes

\begin{tabular}{cllcc}
\hline Docente & Función (titularidad) & Sexo & $\begin{array}{c}\text { Edad } \\
\text { (años) }\end{array}$ & $\begin{array}{c}\text { Antigüedad en la } \\
\text { escuela (en años) }\end{array}$ \\
\hline D1 & 1ro de primaria & Mujer & 35 & 4 \\
D2 & 2do de primaria & Mujer & 41 & 5 \\
D3 & 3ero de primaria & Mujer & 60 & 8 \\
D4 & 4to de primaria & Mujer & 41 & 1 \\
D5 & 5to de primaria & Mujer & 33 & 2 \\
D6 & 6to de primaria & Mujer & 59 & 10 \\
D7 & Computación & Mujer & 32 & 4 \\
D8 & Educación Física & Mujer & 29 & 1 \\
D9 & Educación Artística & Hombre & 27 & 2 \\
\hline
\end{tabular}

\section{Procedimiento}

El proyecto general fue presentado a la dirección de la escuela, que se mostró interesada en participar, y organizó a su vez la presentación del mismo a docentes y padres de familia, quienes manifestaron su disposición a sumarse al mismo por medio de un consentimiento informado.

La primera fase del proyecto (que no se reporta en este trabajo por no estar dentro de sus objetivos), comprendió el diagnóstico e identificación del alumnado con la condición de doble excepcionalidad.

Posteriormente, como parte de una segunda etapa de trabajo, se desarrollaron entrevistas semiestructuradas con los docentes con el fin de explorar y caracterizar su sensibilización y preparación para detectar y dar atención educativa a los alumnos con doble excepcionalidad (Díaz et al., 2013). Las 
entrevistas se llevaron a cabo con toda la planta docente de primaria y se realizaron en modalidad virtual, dadas las condiciones de la contingencia sanitaria por la pandemia de COVID-19 que caracterizaron este momento. Para tal efecto, se utilizó la plataforma Zoom Vídeo que facilitó la grabación, previamente autorizada, de cada encuentro. Todas las entrevistas se realizaron de manera individual en un periodo de trece días en el mes de mayo de 2020. La duración de las mismas varió considerablemente (como promedio 30 minutos, y un máximo de1h $20 \mathrm{~min}$ ) debido a la flexibilidad que se mantuvo en cada encuentro. Los(as) entrevistados(as) tuvieron la oportunidad de extenderse tanto como les fue necesario y de abordar temas que no fueron considerados en el guion previamente establecido.

Para el análisis de la información se trascribieron todas las entrevistas. Las respuestas se sometieron al proceso de análisis de contenido (Bardin, 2002); se pasaron a una matriz de datos cualitativos, con ayuda de la cual se procedió a la codificación, es decir, a la identificación de categorías emergentes, así como de categorías previamente establecidas para procesar la información. Finalmente, el contenido de las respuestas de los docentes se organizó a partir de los siguientes temas o categorías: a) identificación de los(las) estudiantes en función de su AC y del TDAH, b) autoevaluación sobre su conocimiento y dominio de estrategias sobre AC/TDAH, c) percepción de sus fortalezas y áreas de oportunidad, d) percepción de necesidades de formación y/o preparación en temas de doble excepcionalidad. La comparación y contrastación entre las categorías y respuestas de los diferentes participantes, permitió el procesamiento e interpretación de los datos, a partir de las frecuencias de aparición de las mismas a nivel individual y grupal.

\section{Resultados}

A partir del análisis de contenido realizado se establecieron tres categorías: 1. Conocimiento del alumno, 2. Caracterización de la doble excepcionalidad y 3. Autoevaluación docente. Cada categoría contiene subcategorías que se presentan en las Tablas 2, 3 y 4 .

La Tabla 2 sistematiza las respuestas de los maestros en relación al nivel de conocimiento que poseen de los alumnos. En esta categoría se definieron 4 subcategorías: Tiempo de conocer al alumno, Relación con el alumno, Percepciones de los docentes respecto a los alumnos y Necesidades educativas del grupo doblemente excepcional. En la tabla se presentan igualmente los aspectos más relevantes que emergieron del análisis de su contenido. 
Tabla 2

Dimensiones que reflejan el conocimiento que tiene el docente del alumno

\begin{tabular}{|c|c|c|}
\hline Subcategorías & Ejemplos de respuestas & $\begin{array}{c}\text { Aspectos relevantes } \\
\text { emergentes }\end{array}$ \\
\hline $\begin{array}{l}\text { Tiempo de } \\
\text { conocer al } \\
\text { alumno }\end{array}$ & $\begin{array}{l}\text { “...del inicio de este ciclo escolar } 2020 . ” \\
\text { “...aproximadamente como dos años.” } \\
\text { “...al alumno de } 5^{\circ} \text { desde hace tres años, al } \\
\text { alumno de } 6^{\circ} \text { unos ocho meses.” }\end{array}$ & Diferentes tiempos \\
\hline $\begin{array}{l}\text { Relación con el } \\
\text { alumno }\end{array}$ & $\begin{array}{l}\text { “...con el alumno de primer año muy bien, o sea } \\
\text { nunca hubo una dificultad, nos llevamos muy } \\
\text { bien, es una relación afectiva.” } \\
\text { “...siempre tuve mucha afinidad y empatía con él, } \\
\text { desde el inicio se mostró abierto a tener una } \\
\text { relación conmigo.” } \\
\text { “... relación que tenemos es amena, pero al inicio } \\
\text { había como poca confianza.” } \\
\text { “...en un inicio teníamos como dificultades justo } \\
\text { por su condición, fue un poco irrespetuoso, pero } \\
\text { creo que hemos tenido la oportunidad de } \\
\text { ganarnos su confianza.” } \\
\text { “...me ha costado mucho trabajo conocerlo, es un } \\
\text { poco lejana.” }\end{array}$ & $\begin{array}{l}\text { Relaciones } \\
\text { contradictorias, } \\
\text { fluctuantes de } \\
\text { buenas, cercanas, a } \\
\text { lejanas. } \\
\text { Confianza y empatía } \\
\text { se valoran } \\
\text { positivamente por el } \\
\text { docente, mientras } \\
\text { que las conductas } \\
\text { disruptivas, se } \\
\text { pueden interpretar } \\
\text { como faltas de } \\
\text { respeto. }\end{array}$ \\
\hline $\begin{array}{l}\text { Percepciones } \\
\text { respecto al } \\
\text { alumno }\end{array}$ & $\begin{array}{l}\text { “...me parece muy brillante, muy brillante, muy } \\
\text { inteligente, pero también inquieto, muy } \\
\text { inquieto” } \\
\text { “...es muy inteligente, muy hábil, tiene muchas } \\
\text { destrezas, pero como que tiene mucha } \\
\text { inseguridad y desconfianza de lo que él mismo } \\
\text { sabe” } \\
\text { “...es muy listo, cuando le digo quién no termina } \\
\text { no sale, ¿qué crees? Lo hace, entonces ahí está la } \\
\text { dualidad: ¿puedes o no puedes?” } \\
\text { “...tiene como esa hambre de querer aprender, se } \\
\text { esfuerza cuando uno le hace ver en qué cosas } \\
\text { puede mejorar y se enfoca en ello” }\end{array}$ & $\begin{array}{l}\text { Simultaneidad de } \\
\text { características que } \\
\text { apuntan al potencial } \\
\text { (fundamentalmente } \\
\text { respecto a } \\
\text { aprendizaje) y al } \\
\text { déficit (inseguridad, } \\
\text { dificultad en } \\
\text { relaciones, } \\
\text { problemas de } \\
\text { atención) }\end{array}$ \\
\hline
\end{tabular}


“...es muy disperso, podríamos estar dando la

clase y explicando en el pizarrón y está

distraído"

“....es un niño muy penoso, muy introvertido y es

un poquito renuente a seguir instrucciones."

Necesidades educativas “...me tengo que sentar con él, requiere acompañamiento todo el tiempo" “...ponerlo muy cerca de mí y hacer que participe preguntándole cosas para que no pierda la atención, porque si me olvido un poco de él, su cabeza ya está en otro lado."

“...noto que cuando le reconoces sus logros, se motiva y lo hace mucho mejor, es un niño que necesita reconocimiento de sus acciones" "...reconocer sus trabajos, que lo haya hecho bien, escuchar palabras de reconocimiento" "saberlo escuchar, comprender su situación también ayuda" “...no lo puedo poner ni cerca de mis ventanas del lado izquierdo ni cerca de mis puertas del lado derecho, porque seguro se me va a distraer" “...le gustan mucho los retos, que el ejercicio lo rete, que no esté tan fácil como de -ah ya lo entendí-, por ello busco actividades desafiantes" "...trabajar en pares, cuando se le sienta con un compañero que suele ser exitoso, como que eso lo motiva a hacer las cosas bien" “...trabajar los procedimientos, se saben tan capaces, tan que lo saben todo del tema, que piensan que no es necesario pasar por el procedimiento" “...yo creo que le serviría estar en una clase más avanzada, porque entendería bastantes cosas, creo que estaría más tranquilo porque ya tendría más retos"
Necesidades de acompañamiento y de control y regulación externos, como déficit, y reconocimiento de posibilidades de trabajo con estrategias cooperativas, o de implementar desafíos para captar su atención, como potencial.
En general, las respuestas muestran la diversidad de posibilidades que la literatura reconoce en los perfiles de los alumnos doblemente excepcionales. En el primer caso, la subcategoría se refiere a la relación que el docente establece con el alumno, pues es un indicador del nivel de sensibilización que ha desarrollado para 
identificar los rasgos diferenciales. Es significativo el contraste entre respuestas brindadas por los diferentes docentes; por un lado, se describe la relación con el alumno como lejana y con problemas de confianza mientras que, de manera opuesta, se describen relaciones de empatía y disposición. Pese al contraste, la confianza y la empatía son valoradas positivamente por los docentes.

La investigación en altas capacidades (Martínez, 2013), señala que la detección de la excepcionalidad intelectual, por parte del maestro, suele activar mecanismos de defensa que permiten reducir la ansiedad que genera no tener parámetros claros de referencia; esto, a diferencia de la identificación de la discapacidad, en la que se activan mecanismos que priorizan la función de soporte y cuidado. En el caso de los alumnos con doble excepcionalidad, la ansiedad puede ser aún mayor debido a la contradicción entre los rasgos diferenciales, dando lugar así, a posiciones negativas (como la negación de las altas capacidades, la confusión de los rasgos con problemáticas emocionales, posturas rígidas, entre otras) y en general, a una actitud que puede obstaculizar inclusive la propia intervención. Desde esta lógica, resulta necesario conocer la percepción del docente respecto al alumno.

La segunda subcategoría comprende las percepciones de los docentes. En ellas es posible observar respuestas que reflejan el reconocimiento de las dificultades atencionales, así como las dificultades sociales y de integración. Por otra parte, resultan especialmente reveladoras las respuestas que, de alguna manera, visibilizan la paradoja de la doble excepcionalidad. Dicha identificación representa un parámetro de la detección de las necesidades educativas, es decir, si los maestros logran reconocer que se trata de alumnos sumamente inteligentes con problemáticas en el trabajo escolar, es posible que visualicen algunas condiciones qua favorecen o no, el desempeño académico.

De lo anterior, se desprende la relevancia de considerar la detección de las necesidades educativas como una pauta que permitiría una intervención más adecuada por parte de los docentes. En esta subcategoría se evidencia la necesidad de brindar a este grupo de alumnos, un acompañamiento y supervisión constante, la importancia de reconocer sus logros como factor de motivación, la pertinencia de evitar distractores y favorecer el reto intelectual, la conveniencia de solicitar trabajo en equipo (específicamente en parejas) y la trascendencia de formar sus hábitos escolares. Adicionalmente, se asume como destacable la respuesta que indica la necesidad de una clase avanzada para promover el potencial.

En la segunda categoría se agrupan las dimensiones que están orientadas a la caracterización psicopedagógica del alumno por parte del docente. La Tabla 3 muestra algunas de las respuestas de los maestros organizadas en 6 subcategorías: Habilidades académicas, Habilidades cognitivas, Dificultades académicas, Dificultades cognitivas, Interacción con compañeros y Conductas disruptivas. 
Tabla 3

Principales características que se derivan del potencial y del déficit (doble excepcionalidad)

\begin{tabular}{|c|c|c|}
\hline Subcategorías & Ejemplos de respuesta & $\begin{array}{c}\text { Aspectos relevantes } \\
\text { emergentes }\end{array}$ \\
\hline $\begin{array}{l}\text { Habilidades } \\
\text { académicas }\end{array}$ & $\begin{array}{l}\text { “...él ya va como un paso adelante, ya lo dedujo, } \\
\text { entonces en matemáticas y en exposición o } \\
\text { expresión verbal, creo que es donde destaca" } \\
\text { “...es muy bueno para resolver problemas y } \\
\text { realizar operaciones aritméticas, destaca en el } \\
\text { área de matemáticas" } \\
\text { "...le encanta leer y hablar de libros, te habla } \\
\text { mucho de los libros, de la historia, es un gran } \\
\text { lector y su nivel de comprensión lectora es alto" } \\
\text { “...es muy bueno exponiendo, todo lo que tiene } \\
\text { que ver con la explicación verbal se le facilita" } \\
\text { "es muy participativo, se involucra en las } \\
\text { diferentes dinámicas, ya sean grupales o } \\
\text { individuales y siempre responde o da su } \\
\text { opinión” } \\
\text { “...el nivel de comunicación que tiene es como si } \\
\text { estuviera hablando con un adulto, se expresa } \\
\text { como un adulto, te ocupa vocabulario de adulto" } \\
\text { "...son muy creativos porque incluso llegan a } \\
\text { hacer cosas que yo no les digo, pero lo hacen } \\
\text { bastante bien” }\end{array}$ & $\begin{array}{l}\text { Reconocimiento del } \\
\text { potencial de } \\
\text { aprendizaje, de } \\
\text { habilidades y } \\
\text { competencias } \\
\text { específicas, es decir, } \\
\text { de sus fortalezas en } \\
\text { el terreno académico. }\end{array}$ \\
\hline $\begin{array}{l}\text { Habilidades } \\
\text { cognitivas }\end{array}$ & $\begin{array}{l}\text { “...creo que su área más fuerte es el análisis, el } \\
\text { análisis de las cosas lo hace muy bien” } \\
\text { “...logra identificar varias cosas que me he dado } \\
\text { cuenta, que los demás no, pone especial atención } \\
\text { en los detalles” } \\
\text { “...su lógica es bastante buena, tanto que de } \\
\text { pronto algo que todavía no explico, él lo } \\
\text { resuelve, si esto es así, esto podría ser de esta } \\
\text { otra forma” } \\
\text { “...me da la impresión de que su mente va muy } \\
\text { rápido, más rápido de lo que habla, entiende las } \\
\text { cosas muy rápido" } \\
\text { “...tiene muy buena memoria, cuando retomamos } \\
\text { otros temas, vuelve a redactar perfectamente } \\
\text { bien lo que se había platicado” }\end{array}$ & $\begin{array}{l}\text { Reconocimiento de } \\
\text { algunas de las } \\
\text { características } \\
\text { cognitivas } \\
\text { significativas: } \\
\text { memoria, } \\
\text { pensamiento, } \\
\text { velocidad del } \\
\text { procesamiento de } \\
\text { información, } \\
\text { independencia } \\
\text { cognitiva, curiosidad. }\end{array}$ \\
\hline
\end{tabular}


“...esta habilidad de la escucha como muy desarrollada, comprenden auditivamente, parecen distraídos, pero están escuchando". "...ellos solitos buscan a aprender, investigan lo que les interesa, como que tienen la necesidad de saber cosas que otros no"

Dificultades académicas

Dificultades cognitivas “...se le ha dificultado consolidar la lectoescritura, apenas logra leer algunas sílabas" “...se le dificulta la parte del cálculo, por ejemplo, de repente no logra realizar una suma sencilla, se pierde"

"...la estructura escrita, no la tiene desarrollada, puede escribir arriba, desfasarse del renglón, no tiene un orden y a veces su letra es ilegible" “...le cuesta muchísimo la escritura más que la lectura y se resiste a escribir, es como si fuera una tortura"

"...se le dificulta terminar en tiempo y forma sus trabajos o bien, no los entrega"

"...si a ellos no les interesa el tema, la verdad es que no ponen atención, o sea, buscan cualquier pretexto para salirse"

“...ambos alumnos coinciden en la falta de atención y concentración"

“...fácilmente se distrae, entonces si hay que encontrar la manera de regresarlo y hacerlo trabajar, mmm si porque fácilmente se distraen" “...yo creo que esa es su mayor dificultad, el seguimiento de instrucciones, el seguir las reglas, no sigue indicaciones"

“...él te puede estar jugando todo el tiempo, no deja de moverse, hace ruidos, se pone a cantar, pareciera que no controla su cuerpo" “...se le olvida todo, todo el tiempo, hacer tareas, entregar proyectos, traer materiales y constantemente pierde sus objetos escolares" “...la organización y la planeación, sus cosas siempre están desordenadas y no tiene una secuencia para hacer las cosas"
Detección de

dificultades en torno al cálculo y lectoescritura, así como a la posibilidad de trabajo concentrado en tareas que no son de su interés. En la mayoría de los casos, se observa especial resistencia a la escritura.

Detección de dificultades en torno a los procesos de atención, la memoria operativa y las funciones ejecutivas relacionadas con planificación y organización Se observan rasgos que caracterizan la hiperactividadimpulsividad 


\begin{tabular}{|c|c|c|}
\hline & $\begin{array}{l}\text { “...ambos son super sociables, son muy bien } \\
\text { aceptados y queridos, los aprecian mucho, son } \\
\text { de hecho líderes” } \\
\text { “...tardó yo creo que como } 3 \text { semanas en } \\
\text { adaptarse y poder lograr una buena } \\
\text { comunicación dentro del grupo" } \\
\text { “...le cuesta mucho trabajo integrarse al grupo, } \\
\text { frecuentemente se aísla y busca estar solo, pero } \\
\text { ya convive con algunos compañeros” } \\
\text { "...se relaciona un poquito más con las niñas que } \\
\text { con los niños, sobre todo con las niñas que } \\
\text { sobresalen en la parte académica” } \\
\text { “...cuando algo no le gusta, empieza a ser huraño } \\
\text { ¿no? empieza a enojarse e irritarse con los } \\
\text { demás" } \\
\text { “...mayor dificultad es la falta de empatía, cree } \\
\text { que todos piensan como él piensa y que todos } \\
\text { sienten como él siente, entonces dice y hace } \\
\text { cosas que no considera ofensivas, pero que } \\
\text { dañan a los demás” } \\
\text { “...suele imponer mucho lo que él piensa, lo que } \\
\text { él quiere, esto no le genera como buenas } \\
\text { relaciones, no muchos se juntan con él” }\end{array}$ & $\begin{array}{l}\text { Percepción variable } \\
\text { de la interacción y } \\
\text { habilidades sociales y } \\
\text { emocionales, lo cual } \\
\text { muestra la diversidad } \\
\text { respecto a esta área, } \\
\text { sin embargo, se } \\
\text { observa un mayor } \\
\text { énfasis en las } \\
\text { dificultades de } \\
\text { interacción e } \\
\text { integración. }\end{array}$ \\
\hline $\begin{array}{l}\text { Conductas } \\
\text { disruptivas }\end{array}$ & $\begin{array}{l}\text { “...tiene tratos irrespetuosos, a veces utiliza } \\
\text { malas palabras y es grosero" } \\
\text { “...no escucha las indicaciones y no le importa, no } \\
\text { respeta límites" } \\
\text { "...en algunos momentos ha llegado a ser muy } \\
\text { agresivo, impulsivo y explosivo, no controla su } \\
\text { enojo y frustración” } \\
\text { “...ellos son los que, de cierta forma, incitan a los } \\
\text { demás a crear desorden. Poco a poco, pueden } \\
\text { detener por completo una clase” } \\
\text { "...esto de no poder controlar como las malas } \\
\text { palabras y los comentarios negativos, no lo } \\
\text { puede evitar" } \\
\text { "...ha tenido muchos problemas, reportes y se le } \\
\text { ha sancionado con suspensiones precisamente } \\
\text { porque se ha burlado de sus compañeras” }\end{array}$ & $\begin{array}{l}\text { Detección de } \\
\text { conductas en torno a } \\
\text { la impulsividad, la } \\
\text { falta de habilidades } \\
\text { de autorregulación, y } \\
\text { dificultades en } \\
\text { establecimiento de } \\
\text { límites. }\end{array}$ \\
\hline
\end{tabular}

\section{Conductas} malas palabras y es grosero" respeta límites"

“...en algunos momentos ha llegado a ser muy agresivo, impulsivo y explosivo, no controla su enojo y frustración"

...ellos son los que, de cierta forma, incitan a los detener por completo una clase" palabras y los comentarios negativos, no lo puede evitar" "...ha tenido muchos problemas, reportes y se le porque se ha burlado de sus compañeras"
Detección de conductas en torno a falta de habilidades dificultades en establecimiento de límites. 
Las respuestas sistematizadas en la Tabla 3, demuestran que los participantes reconocen algunas de las características que se derivan de la presencia de altas capacidades y del TDAH. Es posible inferir que su experiencia frente a grupos de alumnos les permite reconocer parcialmente los rasgos diferenciales de ambas condiciones; sin embargo, no identifican los rasgos que pueden ser considerados más significativos, como pueden ser la perseverancia en temas de su interés (hiperfocalización) o la dificultad para mantenerse atentos específicamente en actividades rutinarias. Además, no se emitieron respuestas relacionadas con el estado emocional o bien, con las características del autoconcepto del alumnado; si bien es cierto que se refirieron a algunos de los niños como líderes, no plantearon cómo se ven a sí mismos o cómo visualizan sus habilidades o dificultades.

Desde estos planteamientos, resulta evidente la necesidad y pertinencia de brindar a los docentes una preparación que permita, no solamente ampliar los conocimientos que poseen sobre las características de los alumnos doblemente excepcionales, sino también, la fundamentación teórica que explica la complejidad de la simultaneidad, pues en varias respuestas, los participantes manifestaron ideas que asocian las características diferenciales a la voluntad del niño, a su deseo de querer o no querer hacer las cosas.

Finalmente, la tercera categoría analizada se centra en la evaluación que los maestros hicieron de su propio desempeño. La Tabla 4 destaca sus respuestas en torno a la reflexión de sus Fortalezas, Dificultades y Necesidades de formación que son las 3 subcategorías que integran la última categoría.

\section{Tabla 4}

Contenidos de la autoevaluación del docente: Fortalezas y necesidades de formación

\begin{tabular}{|c|c|c|}
\hline Subcategorías & Ejemplos de respuesta & $\begin{array}{c}\text { Aspectos relevantes } \\
\text { emergentes }\end{array}$ \\
\hline $\begin{array}{l}\text { Fortalezas del } \\
\text { docente }\end{array}$ & $\begin{array}{l}\text { “...se me facilita implementar material lúdico que } \\
\text { sea llamativo para ellos y el cual puedan } \\
\text { manipular" } \\
\text { “...yo soy muy empática, me gusta este tipo de } \\
\text { niños, hablo mucho con ellos y los escucho, busco } \\
\text { una buena comunicación” } \\
\text { “...la paciencia, soy super paciente, no llego como a } \\
\text { desesperarme, a ese punto de ah ya no sé qué } \\
\text { hacer” }\end{array}$ & $\begin{array}{l}\text { Percepción y } \\
\text { autovaloración de } \\
\text { competencias } \\
\text { didácticas, } \\
\text { personales, y } \\
\text { socioemocionales. }\end{array}$ \\
\hline
\end{tabular}


“...podría ser en aquello que permite mejorar su conducta, dialogando con ellos, brindándoles ejemplos, haciéndoles ver sus logros" “...cuando estoy pensando en lo que voy a enseñar al siguiente día, lo que más se me facilita es pensar en las actividades que van a motivarlos" “...trabajar con mi mancuerna...trabajamos a la par, en el momento que yo quiero decirles algo, ella está conmigo" “...trabajar en equipo, buscar el apoyo de otros docentes para poder realizar diversas adecuaciones" “...estar en constante capacitación, en el verano tomé dos diplomados en el centro de maestros para dar clases de matemáticas"

Dificultades del docente “...no sé cómo hacerle entender que a veces lo que él piensa no siempre lo va a llevar a lo correcto" “...tengo que ver su letra, revisar ortografía, caligrafía, esta es la parte que me cuesta trabajo que entiendan que tienen que escribir, porque ellos creen que, con solo saberlo ya, no es necesario que escriban" “...la paciencia, no soy totalmente paciente cuando los alumnos presentan estas características" “...cómo acaparar su atención, cómo lograr que te hagan caso.... veces si me cuesta mucho trabajo y me siento muy frustrada"

“...cuando tengo un alumno así, no tengo respuestas claras, intento resolver en el momento, conforme todo va surgiendo y voy probando ¿no? y equivocándome, hasta que bueno, logro ver algo que funciona"

“...mantener el ritmo de una clase y lograr los objetivos es muy difícil cuando tienes un alumno así, se me dificulta, es como: me interrumpiste por esta situación y ahora tengo que retomar o volver a empezar"

“...ellos son los que, de cierta forma, incitan a los demás a crear desorden poco a poco, porque como posibilidad de trabajo en equipo y autosuperación.

Autovaloración del insuficiente desarrollo de habilidades didácticas (organización y control del proceso de enseñanzaaprendizaje) para responder a este tipo de alumnado. 
ya acabaron están desesperados por otra cosa, eso es lo que se me dificulta de pronto". "...digo nunca he gritado mucho, menos lo he ofendido, pero si me molestan las constantes distracciones que genera, puedo perder el control de una clase, eso se me complica, cómo mantener el control de la clase"

Necesidades de formación “...me siento totalmente desarmada, no sé cómo hacer para motivarlo, he intentado muchas cosas y no lo veo interesado en las clases" "quiero saber cómo hacer para tener evidencias de su conocimiento, sin tener que solicitar tanta escritura, eso es lo más difícil" “...no me siento como preparada a veces, lo que el presenta, va más allá de lo que te enseñan en la carrera"

“...cuando muestran hiperactividad, si me encantaría saber una técnica o técnicas para poder apoyar a los chicos"

“...estrategias que me permitan llamar su atención y que se le facilite mantenerse concentrado, que su aprendizaje sea significativo"

“...considero que sí necesito un entrenamiento adicional para saber cómo dar un apoyo completo" “...gustaría saber la teoría, cómo entender ya desde el punto de vista de la educación, cómo funcionan estos niños" “...pues me gustarían técnicas, o sea que, si te llega un alumno así, yo saber rápido, de bote pronto ¡ah tengo que hacer esto!"

“...saber cómo ayudarle ¿no? qué estrategias, que técnicas hay, saber qué es lo que provoca esta situación ¿no?, qué limites se pueden poner, todo va enfocado a cómo ayudarles"
Necesidades

articuladas en torno a estrategias

pedagógicas de naturaleza adaptativas, flexibilidad en las mismas, dominio teórico sobre el tema. Necesidades relativas al trabajo con la atención, motivación y apoyo al desarrollo integral.
En esta categoría, un rasgo distintivo de las respuestas fue la apertura para reconocer las dificultades, las situaciones negativas y, sobre todo, la conciencia de sus propias limitaciones como profesionales de la educación. Aceptar la carga emocional de 
sus actuaciones, es una primera fase en el proceso de analizar sus propias actitudes como barreras o detonantes del potencial del alumnado doblemente excepcional.

\section{Discusión}

El presente estudio estuvo dirigido a explorar la sensibilización y preparación de los docentes para la atención educativa al alumnado con doble excepcionalidad en una escuela primaria.

El análisis de los resultados obtenidos permite destacar algunos aspectos relevantes en el grupo de docentes de una escuela primaria. Los resultados del diagnóstico de la sensibilización y preparación de los maestros para detectar y dar atención educativa a los alumnos con doble excepcionalidad, fueron analizados a partir de tres categorías aspectos que son esenciales para la organización de cualquier intervención por parte del profesorado: a) el conocimiento del alumno, y la particular sensibilidad ante sus necesidades y comportamientos típicos; b) la posibilidad de identificar los aspectos relevantes de su condición de alta potencialidad, por una parte, $y$ de desventaja, por la otra, es decir, las posibilidades de caracterización de la doble excepcionalidad, y c) la posibilidad de realizar una autoevaluación de sus propias posibilidades como docente para dar una atención a este alumnado, así como de la motivación para apropiarse de nuevas herramientas y recursos para poder hacer, transformando así su propio ejercicio y desempeño profesional. Estas categorías, como también se ha comprobado en estudios previos (Conejeros et al, 2018) demostraron poseer un alto valor descriptivo para comprender los focos de las carencias, preocupaciones y fortalezas de los docentes en este campo.

Como se ha enfatizado en diversas investigaciones, un proceso esencial en el profesorado es la toma de conciencia que se requiere para comprender la complejidad que representa la doble excepcionalidad (Conejeros et al, 2018; Gómez et al., 2016). En este sentido, el desarrollo de una postura autocrítica en el docente, este es un proceso clave para lograr un cambio de perspectiva que se traduzca en un cambio actitudinal a favor, no solo de los alumnos con altas capacidades y/o doblemente excepcionales, sino a favor de su propio ejercicio profesional. Esto ocurre, en general, cuando se analizan las motivaciones y actitudes del profesorado para implementar intervenciones educativas con el alumnado (Castellanos, 2014; Martínez, 2013).

Asimismo, el análisis del diagnóstico realizado a los docentes, como se corrobora en revisiones de investigaciones realizadas por autores como Álvarez-Cárdenas et al. (2019) evidencia la vulnerabilidad y frustración que experimentan ante la falta de recursos teórico - metodológicos para intervenir de manera satisfactoria frente a alumnos con perfiles diferenciales, que incluyen habilidades cognitivas destacadas, demuestra que resulta imprescindible formarlos en la detección y atención de los alumnos con altas capacidades con o sin $\mathrm{TDAH}$, con el objetivo de posibilitar que respondan favorablemente a las necesidades educativas de estos alumnos. 
Los resultados obtenidos revelan que el escenario de enmascaramiento más frecuente es aquel en el que se invisibiliza el potencial o se dificulta la valoración plena de ese potencial, obstaculizando la posibilidad de detonar las altas capacidades. Por lo tanto, se considera necesario, persistir en la iniciativa de promover la formación de los docentes en detección y atención de las altas capacidades intelectuales pues, como se evidenció, puede pasar desapercibida. En línea con lo planteado por Castellanos (2014), la preparación de los docentes es una pieza fundamental en la promoción del potencial.

La falta de recursos personales y emocionales que se manifestó en el diagnóstico docente, alude a la inseguridad que les genera responder favorablemente a las necesidades de alumnos que, por la complejidad de la simultaneidad, observan perfiles contradictorios y de difícil categorización, resulta imprescindible entonces, empoderar su práctica profesional mediante el empleo de una metodología que promueva la participación activa de los involucrados en un proceso de cambio que se caracteriza por fortalecer la conciencia colectiva, por la promoción de una comprensión crítica y por la gestión de decisiones que posibilitan una acción transformadora, es por ello que se asume como pertinente, la investigación - acción participativa como el diseño que dará estructura a la intervención.

Los docentes participantes en la investigación manifestaron parcialmente un reconocimiento de algunas de las características diferenciales de este tipo de alumnado. La adquisición de habilidades para la identificación resulta necesaria para la caracterización del perfil de los alumnos, así como para la detección de sus necesidades educativas especiales.

\section{Conclusión}

En general, tanto a nivel internacional como en particular, en el contexto latinoamericano, la investigación e intervención en el campo de la doble excepcionalidad en aún un espacio abierto de búsqueda, que muestra múltiples lagunas, necesidades y oportunidades de desarrollo. Una de las direcciones básicas de desarrollo tiene que ver con la preparación del profesorado para hacer frente a la implementación de estrategias y programas de detección y atención educativa a esta población de estudiantes. Una de las tareas más importantes en este sentido es documentar las necesidades de formación sentidas por los docentes, explorar sus propias perspectivas, dar "voz" a sus preocupaciones y temores en este sentido.

En este estudio, las principales necesidades que se hicieron evidentes en el profesorado están relacionadas con:

1. La necesidad de la apropiación de herramientas y recursos para la creación de propuestas de intervención basadas en las fortalezas de los estudiantes.

2. El desarrollo de destrezas que favorecen la estimulación del potencial, propiciando un 
ambiente desarrollador de todos los alumnos.

3. La preparación que les permita responder favorablemente al reto de brindar una atención diferenciada a los alumnos que presenten una de las tres posibilidades de la doble excepcionalidad: Alta Capacidad, TDAH y por supuesto, la combinación de ambas.

\section{Referencias}

Álvarez-Cárdenas, F., Peñaherrera-Vélez, M., Arévalo-Proaño, C., Dávila, Y. \& Vélez-Calvo, X. (2019). Altas capacidades y TDAH: una doble excepcionalidad poco abordada. International Journal of Developmental and Educational Psychology. 5(1), 417-428. http://doi.org/10.17060/ijodaep.2 019.n1.v5.1621

Bardin, L. (2002). El análisis de contenido. Madrid: Ediciones Akal. https://books.google.com.pe/book s?id=IvhoTqll_EQC\&printsec=front cover $\& \mathrm{hl}=\mathrm{es} \# \mathrm{v}=$ onepage $\& \mathrm{q} \& \mathrm{f}=\mathrm{fals}$ $\mathrm{e}$

Baum, S. (1989). Gifted but learning disabled: A puzzling paradox. Preventing School Failure: Alternative Education for Children and Youth, 34(1), 11-14. https://doi.org/10.1080/1045988 X.1989.9944546

Beckley, D. (1998). Gifted and learning disabled: Twice exceptional
Como sostienen Conejeros et al. (2018), el desarrollo de la investigación que potencie el rol de los docentes, su capacitación desde pedagogías positivas y desde el dominio de posibilidades de enriquecimiento curricular y de orientación psicopedagógica para el alumnado con doble excepcionalidad es esencial, como parte importante del logro de una educación inclusiva.

students.

http://www.gifted.uconn.edu/nrcg t/newsletter/spring98/sprng984.h tml

Bianco, N. \& Leech, N. L. (2010). TwiceExceptional Learners: Effects of Teachers Preparation and Disability Labels on Gifted Referral. Teacher Education and Special Education, $33(4)$

319-334. https://doi.org/10.1177/0888406 409356392

Castellanos, D. (2014). Sensibilización y preparación de docentes: piezas clave en la atención educativa a los alumnos con altas capacidades y talentos. En A. Bazán \& D. Castellanos. (Eds.), La Psicología en la Educación, Contextos de Aprendizaje e Investigación (pp. 147-177). Editorial UAEM-Plaza y Valdés Editores.

Conejeros, M. L., Gómez, M., Sandoval, K., \& Cáceres-Serrano, P. A. (2018). Aportes a la comprensión de la 
doble excepcionalidad: Alta capacidad con trastorno por déficit de atención y alta capacidad con trastorno del espectro autista. Revista Educación,42(2), 3-22. https://doi.org/10.15517/revedu.v $42 \mathrm{i} 2.25430$

Conejeros-Solar, M. L., Sandoval-Rodríguez, K., Cáceres-Serrano, P. \& GómezArizaga M. P. (2018). Doble excepcionalidad: Manual de identificación y orientaciones psicoeducativas. Dirigido a profesores de niños, niñas $y$ adolescentes doblemente excepcionales. Pontificia Universidad Católica de Valparaíso.

Díaz, L., Torruco, U., Martínez, M., y Varela, M. (2013). La entrevista, recurso flexible y dinámico. Investigación en educación médica, 2(7), 162-167. http://www.scielo.org.mx/scielo.p hp?script=sci_arttext\&pid=S200750572013000300009\&lng=es\&tlng $=\mathrm{es}$

Flick, U. (2012). Introducción a la investigación cualitativa. Morata.

García, A. (2013). Trastornos del Aprendizaje y conducta en situaciones de alta capacidad intelectual.

http://www.neuropediatriaytdah.c om/wpcontent/uploads/2018/02/LIBROALTA-CAPACIDAD-INTELECTUAL002.pdf

Gómez-Arizaga, M., Conejeros-Solar, M., Sandoval-Rodríguez, K., \& Armijo-
Solís, S. (2016). Doble excepcionalidad: Análisis exploratorio de experiencias $\mathrm{y}$ autoimagen en estudiantes chilenos. Revista de Psicología, 34(1), 5-37. https://doi.org/10.18800/psico.20 1601.001

Kvale, S. (2008). Las entrevistas en la investigación cualitativa. Morata.

Luque-Parra, D., Luque-Rojas, M., \& Hernández, R. (2017). Altas capacidades intelectuales $\mathrm{y}$ trastorno de déficit de atención con hiperactividad: a propósito de un caso. Pontificia Universidad Católica de Valparaíso, Perspectiva Educacional. Formación de Profesores, 56, 164-182. https://doi.org/10.4151/0718972 9

Martínez, M. (2013). Altas capacidades intelectuales. Modelos teóricos. En M. Martínez y Á. Guirado, (Coord.), Altas capacidades intelectuales. Pautas de actuación, orientación, intervención y evaluación en el periodo escolar (pp. 33-63). Graó.

Pardo de Santayana, R. (2002). Superdotación intelectual y trastorno por déficit de atención e hiperactividad (TDAH). FAISCA Revista de Altas Capacidades, 9, 126135. https://doi.org/10.4151/0718972 9

Pardo de Santayana, R. (2004). Alumnos doblemente excepcionales: 
superdotación intelectual y dificultades de aprendizaje. FAISCA: Revista de Altas Capacidades, 11, 3746.

Pfeiffer, S. (2015). Gifted students with a coexisting disability: The twice exceptional. Florida State University. Department of Educational Psychology and Learning Systems, 32(4), 717-727. https://doi.org/10.1590/0103166X2015000400015

Raven, J., Court, H. \& Raven, J. (2001) Test de Matrices Progresivas: Escalas Coloreada, General y Avanzada. Manual (2da. ed.). TEA Ediciones.

Sevilla, D. R., Martín, M. J. \& Jenaro, C. (2018). Actitud del docente hacia la educación inclusiva y hacia los estudiantes con necesidades educativas especiales. Innovación Educativa, 18(78), 115-142. http://www.scielo.org.mx/scielo.p hp?script=sci_arttext\&pid=S166526732018000300115\&lng=es\&tlng $=\mathrm{es}$.
Valadez, M. D., Aguiñaga, L. A., Morales, J. S., Verche, E. Borges, A. \& Rodríguez, C. J. (2018). Atención e Impulsividad en Niños con Alta Capacidad Intelectual y Niños con TDAH. Electronic Journal of Research in Educational Psychology, 16(3), 503516. https://doi.org/10.25115/ejrep.v1 $6 \mathrm{i} 46.2232$

Wechsler, D. (2007). WISC-IV: Escala de Inteligencia de Wechsler para NiñosIV (2a ed.). TEA Ediciones. 